\title{
Sex Disparities in Access to Surgical Care at a Single Institution in Malawi
}

\author{
Trista D. Reid ${ }^{1,2,4} \cdot$ Sherry M. Wren ${ }^{3} \cdot$ Joanna Grudziak $^{1,2} \cdot$ Rebecca Maine $^{1,2,4}$. \\ Chifundo Kajombo ${ }^{2} \cdot$ Anthony G. Charles ${ }^{1,2,4}$
}

Published online: 25 August 2018

\begin{abstract}
Introduction There is a paucity of data regarding sex-based disparities in surgical care delivery, particularly in lowand middle-income countries. This study sought to determine whether sex disparities are present among patients presenting with surgical conditions in Malawi. Hypothesis compared to men, fewer women present to Kamuzu Central Hospital $(\mathrm{KCH})$ with peritonitis and have longer delays in presentation for definitive care.

Methods This study performs a retrospective analysis of prospectively collected data of all general surgery patients with peritonitis presenting to KCH in Lilongwe, Malawi, from September 2013 to April 2016. Multivariable linear and logistic regressions were used to assess the effect of sex on mortality, length of stay, operative intervention, complications, and time to presentation.

Results Of 462 patients presenting with general surgery conditions and peritonitis, $68.8 \%$ were men and $31.2 \%$ were women. After adjustments, women had significantly higher odds of non-operative management when compared to men (OR 2.17, 95\%CI 1.30-3.62, $P=0.003$ ), delays in presentation (adjusted mean difference $136 \mathrm{~h}, 95 \% \mathrm{CI}$ $100-641, P=0.05$ ), delays to operation (adjusted mean difference 1.91 days, 95\% CI $1.12-3.27, P=0.02$ ), and longer lengths of stay (adjusted mean difference 1.67 days, 95\% CI 1.00-2.80, $P=0.05$ ). There were no differences in complications or in-hospital or Emergency Department mortality.

Conclusion Sex disparities exist within the general surgery population at KCH in Lilongwe, Malawi. Fewer women present with surgical problems, and women experience delays in presentation, longer lengths of stay, and undergo fewer operations. Future studies to determine mortality in the community and driving factors of sex disparities will provide more insight.
\end{abstract}

\section{Introduction}

Trista D. Reid

trista_reid@med.unc.edu

1 Department of Surgery, University of North Carolina- Chapel Hill, 4001 Burnett-Womack Building CB\# 7050, Chapel Hill, NC 27599-7050, USA

2 Department of Surgery, Kamuzu Central Hospital, P.O. Box 149, Lilongwe, Malawi

3 Department of Surgery, Stanford University, 300 Pasteur Drive, Stanford, CA 94304, USA

4 UNC School of Medicine, University of North Carolina, 4008 Burnett Womack Building, Chapel Hill, NC 27599, USA
Sex inequality in health care exists throughout the world, particularly in low- and middle-income countries (LMICs) where women are vulnerable and have less access and control over healthcare decisions than do men [1-4]. In certain regions, the contrast in access to care between men and women is stark: Women cannot seek health care without permission from their husbands [3, 5, 6]. The consequences of disparities in access to surgical care are illustrated in patients with acute abdominal emergencies, where disparities leading to a delay in presentation for an 
emergency can be life-threatening [7]. Highlighting this dilemma, a recent survey of Rwandan households discovered that approximately $30 \%$ of household deaths might have benefited from surgical treatment and prevented mortality [8]. The majority of general surgery conditions are not sex-specific. A recent review of emergency general surgery cases in the USA demonstrated that $46 \%$ of patients were male and 54\% were female [9]. Few investigations have evaluated sex disparities among surgical populations in resource-poor settings [10-16].

This study sought to characterize the prevalence of sexrelated disparities among patients presenting with abdominal surgical emergencies to Kamuzu Central Hospital $(\mathrm{KCH})$ in Lilongwe, Malawi. We hypothesized that disparities in access to surgical care disproportionally affect women, causing delayed presentation and resulting in higher morbidity and mortality.

\section{Methods}

This is a retrospective study of prospectively collected acute care surgery data for all patients who presented to Kamuzu Central Hospital (KCH), Lilongwe, Malawi, between September 2013 and April 2016. KCH is an approximately 1000-bed referral hospital with a catchment of five million people that is the tertiary referral center for eight district hospitals with minimal surgical capabilities. In partnership with $\mathrm{KCH}$, the University of North Carolina (UNC) established a hospital-based acute care general surgery registry to capture disease characteristics and surgical outcomes of this population. The on-site database is kept on password-protected computers and data on the patients consulted on or admitted to the general surgery service are entered weekly by ten trained local clerks hired by UNC.

All patients presenting to the hospital for acute care surgical conditions with peritonitis between September 2013 and April 2016, except those undergoing sex-specific operations (obstetric, gynecologic, and inguinal hernia operations), were included. Trauma patients were also excluded as trauma patients are more likely to be male and men sustaining trauma may have a higher mortality. Peritonitis was defined as generalized abdominal pain with voluntary or involuntary guarding, diagnosed by the admitting clinician. The primary outcome was time to presentation for an acute care surgery condition, defined as hours to presentation to $\mathrm{KCH}$ since patient reported onset of symptoms. This was reported as a continuous variable as mean time in hours.

Secondary outcomes included in-hospital mortality, mortality in the Emergency Department, hospital length of stay in days, operative intervention, time in days from presentation to operative intervention, and postoperative complications. Similar to time to presentation, total hospital length of stay in days was reported as a continuous variable. The variable had a skewed distribution but with a normal distribution of the residuals (errors) thus was used in the models described below. Time to operation was broken down into time to operation in days from presentation to $\mathrm{KCH}$ and in days from presentation to any hospital (to include any transfer time.) These were reported as continuous variables in median days for Table 1 and adjusted models and categorized for Table 2 and Fig. 1 to show distribution. Postoperative complications included wound infection, dehiscence, enterocutaneous fistula, need for re-operation (bleeding, enterotomy, and abscess), and respiratory insufficiency requiring noninvasive or invasive ventilation. Outpatient complications were difficult to track given poor follow-up; thus, the complications were limited to in-hospital complications. Baseline information was collected and reported in Table 1.

Comorbidities that are common in HICs were rare among this patient population; thus, calculating comorbidity indices was not useful. The clerks documented history of tuberculosis, recent malaria, HIV, sickle cell, hypertension, diabetes, known cancer, and mental retardation. The presence or lack of any comorbid condition was used for multivariable analysis. While data were collected on admission vitals, more than half of patients were missing these variables; thus, the study could not reliably use these measures as markers of disease severity. Limiting the cohort to patients presenting with peritonitis was the only way to account for disease severity.

Patient demographics, stratified by sex, were compared using Chi-squared tests. A $P$ value $\leq 0.05$ was considered significant. Wilcoxon-Mann-Whitney test was used for length of stay, and two-variable $t$-test was performed for time to presentation, stratified by sex. Multivariate linear regression was performed for primary and secondary outcomes that were continuous variables, and logistic regression was performed for dichotomous variables. All models adjusted for potential confounders including age, occupation, year of presentation, comorbidities, diagnosis, and surgical intervention. The models for mortality and complications adjusted for delay in presentation and delay in operative intervention, while the model for length of stay adjusted for complications and operative intervention.

All analyses were performed using Stata/MP (Version 12) (Stata Corp, College Station, TX). This study was approved by both the Malawi Ministry of Health and the UNC Institutional Review Board. 


\section{Results}

Overall, 1788 patients presented to $\mathrm{KCH}$ between September 2013 and April 2016 with general surgery problems. Of the 462 patients with peritonitis included in our analysis, $318(68.8 \%)$ were men and $133(31.2 \%)$ were women, Table 1 . The majority of patients who presented to $\mathrm{KCH}$ were transferred from district hospitals $(87.7 \%$ of men and $87.5 \%$ of women), and $61 \%$ of both men and women were from rural settings. The most common employment was blue collar work (45.7\%), which included farming, construction, driving, mechanics and plumbing, restaurant work, and housekeeping. Men were more likely to do blue collar work $(53.8 \%$ vs. $27.8 \%, P<0.0001)$. Men and women had equal percentages of overall comorbidities $(21.4 \%$ of men and $23.6 \%$ of women, $P=0.56)$.

Table 1 General surgery patient characteristics, stratified by sex. $N=462$

\begin{tabular}{|c|c|c|c|}
\hline & $\begin{array}{l}\text { Male } \\
318(68.8 \%)\end{array}$ & $\begin{array}{l}\text { Female } \\
144(31.2 \%)\end{array}$ & $P$ value $^{\mathrm{a}}$ \\
\hline Age, in years, mean $( \pm \mathrm{SD})$ & $34.3( \pm 0.9)$ & $32.7( \pm 1.5)$ & 0.30 \\
\hline \multicolumn{4}{|l|}{ Occupation, $n(\%)$} \\
\hline Child & $1(0.3)$ & $4(2.8)$ & 0.02 \\
\hline Blue collar & $171(53.8)$ & $40(27.8)$ & $<0.0001$ \\
\hline White collar & $52(16.4)$ & $16(11.1)$ & 0.14 \\
\hline Student & $60(18.9)$ & $30(20.8)$ & 0.62 \\
\hline Unemployed & $32(10.1)$ & $23(16.0)$ & 0.07 \\
\hline Housewife & $2(0.6)$ & $31(21.5)$ & $<0.0001$ \\
\hline Transferred from other hospital & $279(87.7)$ & $126(87.5)$ & 0.88 \\
\hline \multicolumn{4}{|l|}{ Domicile location } \\
\hline Urban & $124(39.0)$ & $56(38.9)$ & 0.98 \\
\hline Rural & $194(61.0)$ & $88(61.1)$ & 0.98 \\
\hline \multicolumn{4}{|l|}{ Postoperative diagnosis } \\
\hline Primary peritonitis & $26(8.2)$ & $15(10.4)$ & 0.21 \\
\hline Appendicitis & $83(26.1)$ & $29(20.1)$ & 0.50 \\
\hline Bowel obstruction & $27(8.5)$ & $17(11.8)$ & 0.10 \\
\hline Bowel perforation & $49(15.4)$ & $22(15.3)$ & 0.07 \\
\hline Volvulus & $15(4.7)$ & $4(2.8)$ & 0.47 \\
\hline Ventral hernia & $3(0.9)$ & $2(1.4)$ & 0.56 \\
\hline Gastric perforation & $38(11.9)$ & $3(2.1)$ & 0.002 \\
\hline Other & $7(2.2)$ & $6(4.2)$ & 0.14 \\
\hline \multicolumn{4}{|l|}{ Comorbidities } \\
\hline None & $250(78.6)$ & $110(76.4)$ & 0.56 \\
\hline TB treatment in past & $5(1.6)$ & $0(0.0)$ & 0.13 \\
\hline Malaria in past 2 weeks & $37(11.6)$ & 21 (14.6) & 0.38 \\
\hline Hypertension & $1(0.3)$ & $0(0.0)$ & 0.50 \\
\hline Diabetes & $4(1.3)$ & $0(0.0)$ & 0.18 \\
\hline Known cancer & $1(0.3)$ & $0(0.0)$ & 0.50 \\
\hline Sickle cell & $1(0.3)$ & $0(0.0)$ & 0.50 \\
\hline Mental retardation/delay & $1(0.3)$ & $1(0.7)$ & 0.57 \\
\hline Other & $11(3.5)$ & $9(6.3)$ & 0.17 \\
\hline HIV positive & $5(1.6)$ & $4(2.7)$ & 0.61 \\
\hline Operative intervention & $249(78.3)$ & $98(68.1)$ & 0.02 \\
\hline Intensive care unit stay & $7(2.2)$ & $2(1.4)$ & 0.56 \\
\hline Blood transfusion & $26(8.2)$ & $20(13.9)$ & 0.06 \\
\hline Length of stay in days, median $( \pm \mathrm{SD})$ & $10.1( \pm 0.64)$ & $12.9( \pm 1.5)$ & 0.04 \\
\hline Hours to presentation, mean $( \pm \mathrm{SD})$ & $144( \pm 153)$ & $291( \pm 115)$ & 0.54 \\
\hline
\end{tabular}

$S D$ standard deviation, $T B$ tuberculosis

${ }^{\mathrm{a} P} P$ values $\leq 0.05$ in bold 
Table 2 Distribution of time to operation by sex, $N=328$

Time to operation

Same day, $n(\%)$

Next day

48-72 h

$3-5$ days

6-10 days

$>10$ days

${ }^{\mathrm{a}} P$ values $\leq 0.05$ in bold

\section{Male \\ $236(72.0 \%)$}

$165(69.9)$

$55(23.3)$

8 (3.4)

$4(1.7)$

$3(1.3)$

$1(0.4)$
Female

$92(28.0 \%)$

$50(54.3)$

$30(32.6)$

5 (5.4)

2 (2.2)

3 (3.3)

2 (2.2)
$P$ value $^{\mathrm{a}}$

0.01

0.08

0.39

0.77

0.23

0.14

Fig. 1 Distribution of time to operation by sex

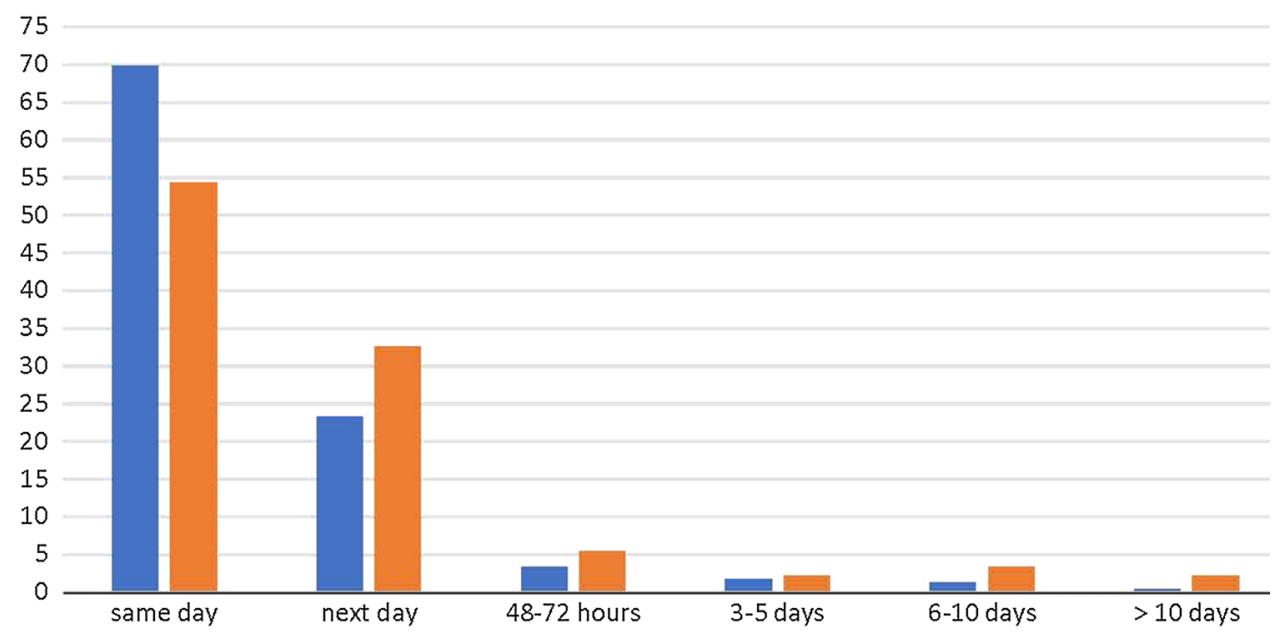

ICU admissions, blood transfusions, and admission and postoperative diagnoses were equally represented among men and women, except for gastric perforations (11.9\% of men vs. $2.1 \%$ of women, $P=0.002$ ).

Time from onset of symptoms to hospital presentation did not differ significantly between men and women in the unadjusted model $(144 \mathrm{~h} \pm 153$ for men and $291 \mathrm{~h} \pm 115$ for women, $P=0.54$.) Before adjustment, women were less likely to undergo operations for peritonitis than were men $(68.1 \%$ vs $78.3 \%, P=0.02)$, had longer times from $\mathrm{KCH}$ arrival to operative intervention, and longer overall lengths of hospital stay (12.9 days vs. 10.1 days, $P=0.04$ ) than did men. Only $54 \%$ of women underwent an operation within $24 \mathrm{~h}$ of presentation compared to $70 \%$ of men $(P=0.01)$, Table 2 , Fig. 1 . Inpatient complications were evenly distributed among women and men; 15 (12.2\%) women and $26(9.2 \%)$ men had a complication, with wound complications being most common, Table 3 .

After adjusting for confounding variables, no differences were found between men and women in terms of in- hospital mortality (OR 1.16, 95\%CI $0.64-2.10, P=0.61$ ) and mortality in the Emergency Department (OR 1.40, 95\%CI $0.22-8.89, P=0.72$ ), Table 3. Additionally, overall complications among men and women did not differ significantly. However, women had significantly delayed time to presentation to $\mathrm{KCH}$, presenting an adjusted mean difference of $136 \mathrm{~h}$ later than men (95\%CI 100-641, $P=0.05$ ). This significance did not persist in time to presentation to any hospital; however, the data for presentation to other facility were missing for $39 \%$ of the patients, regardless of sex. Women had delays in time from presentation to $\mathrm{KCH}$ to operative intervention, undergoing operations an adjusted mean difference of 1.91 days later (95\%CI $1.12-3.27, P=0.02$ ). They had delays in time from presentation to any hospital to operative intervention, undergoing operations an adjusted mean difference of 1.89 days later ( $95 \% \mathrm{CI} 1.12-3.17, P=0.02)$; however, as mentioned above, data were missing for $39 \%$ of patients. Women had longer lengths of stay (adjusted mean difference of 1.67 days, $95 \%$ CI $1.00-2.80, P=0.05$ ) and fewer 
Table 3 Outcomes stratified by sex and adjusted effects of sex on mortality and post-surgical complications, $N=462$

\begin{tabular}{|c|c|c|c|c|}
\hline & \multicolumn{2}{|l|}{ Male female } & \multicolumn{2}{|l|}{ Adjusted $^{\mathrm{a}}$} \\
\hline & $318(68.8 \%)$ & $144(31.2 \%)$ & OR $(95 \% \mathrm{CI})$ & $P$ value ${ }^{\mathrm{b}}$ \\
\hline Death in Emergency Dept & $3(0.9)$ & $3(2.1)$ & $1.40(0.22-8.89)$ & 0.72 \\
\hline Death in hospital & $53(16.7)$ & $26(18.1)$ & $1.16(0.64-2.10)$ & 0.61 \\
\hline Time to presentation to $\mathrm{KCH}$, mean hours $( \pm \mathrm{SD})$ & $144( \pm 153)$ & $291( \pm 115)$ & $136(100-641)^{\mathrm{c}}$ & 0.05 \\
\hline Time to presentation to any hospital, mean hours $( \pm \mathrm{SD})^{\mathrm{d}}$ & $175.2( \pm 67)$ & $\begin{array}{l}116.4 \\
\quad( \pm 27)\end{array}$ & $\begin{aligned}- & 46.3 \\
& (-249-156)^{\mathrm{c}}\end{aligned}$ & 0.65 \\
\hline Time to operating room on arrival to $\mathrm{KCH}$, median days ( $\pm \mathrm{SD}$ ) & $0.6 \pm 3.3$ & $1.3( \pm 2.9)$ & $1.91(1.12-3.27)^{\mathrm{c}}$ & 0.02 \\
\hline $\begin{array}{l}\text { Time to operating room from presentation to any hospital, median days } \\
( \pm \text { SD) }\end{array}$ & $1.6( \pm 3.7)$ & $2.7( \pm 4.1)$ & $1.89(1.12-3.17)^{\mathrm{c}}$ & 0.02 \\
\hline Length of stay, median days ( \pm SD) & $\begin{array}{l}10.1 \\
\quad( \pm 0.64)\end{array}$ & $12.9( \pm 1.5)$ & $1.67(1.00-2.80)^{\mathrm{c}}$ & 0.05 \\
\hline No operative intervention & $69(21.7)$ & $46(31.9)$ & $2.17(1.30-3.62)$ & 0.003 \\
\hline Overall complications & $26(9.2)$ & $15(12.2)$ & $1.20(0.58-2.51)$ & 0.62 \\
\hline Wound infection or dehiscence & $14(4.4)$ & $6(4.2)$ & & \\
\hline Re-operation ${ }^{\mathrm{e}}$ & $5(1.6)$ & $3(2.1)$ & & \\
\hline Respiratory insufficiency & $5(1.6)$ & $4(2.8)$ & & \\
\hline
\end{tabular}

OR odds ratio, CI confidence interval, Dept Department, $S D$ standard deviation

${ }^{a}$ Adjusted for patient age, occupation, year of presentation, comorbidities, presence of peritonitis, surgical intervention, hours to presentation, transfer status, and domicile location

${ }^{\mathrm{b}} P$ values $\leq 0.05$ in bold

${ }^{\mathrm{c}}$ Adjusted mean difference

${ }^{\mathrm{d}}$ Missing variable in $39 \%$ of transfer patients

${ }^{\mathrm{e}}$ Bleeding, enterotomy, abscess

operative interventions when compared to men (OR 2.17, 95\%CI $1.30-3.62, P=0.003$ ). When gastric perforations, which were more common among men, were excluded, the results did not change. When examining the subgroup of patients who underwent surgical management, women continued to have a statistically significant increase in total length of stay.

\section{Discussion}

This study demonstrates that disparities in utilization of surgical care based on sex exist in a subset of patients with surgical conditions and peritonitis at $\mathrm{KCH}$. For acute care, surgical conditions that should have equal representation among men and women, men were overly represented, comprising almost $70 \%$ of all cases. Women were more likely to have delayed presentation to $\mathrm{KCH}$, delayed time to surgical intervention, longer lengths of stay, and less likelihood of surgical intervention. Although there were no differences in in-hospital morbidity and mortality, the smaller number of women presenting for care may represent mortality prior to transfer to our tertiary referral center or in the community.
This study is the first to study sex-based disparities among patients with acute general surgical conditions in sub-Saharan Africa. There have been few studies on sex disparities among surgical patients, with a particular dearth in LMICs. A study of north Indian children presenting for congenital cardiac surgery revealed that only $37.6 \%$ of the children were female [15]. Female children may present less frequently for general hospital conditions in Africa as well; research in Ethiopia and Ghana looking at pediatric admissions and treatment of fever demonstrates different treatment patterns and fewer admission for female patients. $[16,17]$ Studies in Bangladesh, Pakistan, Botswana, and Malawi have demonstrated that women are less likely to seek surgical treatment for cataract surgery, are more likely to present in a delayed fashion, and are more likely to have worse ophthalmologic outcomes than do men [10, 12-14].

In the USA, more robust research has evaluated sexbased outcomes disparities among surgical patients. Female sex has been associated with an increased mortality in burn and pediatric cardiac surgery patients, and higher rates of postoperative complications and mortality following abdominal aortic aneurysm repairs. [11, 18-20]. Women are less likely to receive liver or kidney transplants and are more likely to die on transplant waiting lists [21-24]. 
The specific drivers of sex-based disparities in access to surgical care are not well delineated, and the reasons for the disparities identified in this study are likely multifaceted. Sociocultural norms and religion are two reasons why sex disparities in surgery may exist. Women's roles as caregivers drive them to prioritize their families' needs over their own, or patriarchal social norms lead to difficulties in women being able to express their needs [3]. Sexbased disparities in outcomes may also simply be attributable to a sex-based predilection to the specific disease or underlying biological differences between men and women, as is seen in symptom and treatment patterns in women with acute coronary syndrome [25-29].

Disparities may be partially due to nuanced differences in symptomatology between men and women; women may have a broader differential for abdominal pain that includes gynecologic diseases, leading to potential for delay or alterations in treatment [30].

Socioeconomic factors also contribute to disparities. In Malawi, health care is free; thus, once patients are admitted to the hospital, delays and differences in care related to economic factors would dissipate. However, the effects of indirect costs must be considered as well and may contribute to delays in presentation. Obtaining transportation, leaving the home, or taking time off work to seek health care is costly. If men are traveling to work in areas closer to hospitals, perhaps they are more inclined to utilize health services. Health literacy and local perceptions of modern health care and hospitals may also contribute. A landmark study in Giza, Egypt showed that among Egyptian women, the most significant factor in healthcare utilization was the women's own perceptions about their health and the belief that certain gynecological conditions were normal [5].

Many of these factors likely contributed to the disparities seen at $\mathrm{KCH}$. One observation of this study was that while there was no difference in time to presentation to $\mathrm{KCH}$ among men and women in the unadjusted analysis, time to presentation became significant in the adjusted model. This difference may have been because women were more likely to be housewives with household or child care responsibilities, and adjusting for occupation led to statistical significance.

Interestingly, $87 \%$ of male and female patients were transfers. Delay in transfer may have contributed to delay in presentation. While this delay would still signify a sex disparity, the underlying cause of the disparity might be different. For example, physicians at the district hospital may be culturally biased to place priority on male patients, and real or perceived differences in the differential diagnosis of female and male abdominal pain could influence decisions to transfer. While clinical picture or illness severity may influence operative decision making, similar cultural biases and diagnostic uncertainty may have influenced the observed delay to operative intervention and differences in management of the patients once admitted to $\mathrm{KCH}$.

Misdiagnosis could have affected the clinical course and contributed to the lack of difference in mortality among men and women in spite of delays and disparate operative management. Some gynecological cases may have been inadvertently included that could be treated without operative management, such as pelvic inflammatory disease or tubo-ovarian abscess. Additionally, appendicitis can be treated non-operatively with antibiotics. If more women were being treated with antibiotics only and had appendicitis or a gynecological diagnosis, overall female mortality might have been affected.

Limitations of the study include that this population is a cross section of the country and so may not be generalizable to other regions of the country or other LMICs. The study is hospital-based; therefore, we do not know what morbidity and mortality are being experienced in the community setting.

Unfortunately, 39\% of patients did not have data regarding time to arrival at outside facility; thus, time to presentation to any facility and time to operation from presentation to any facility are difficult to interpret and delay in transfers may have affected presentation. There was not a sufficient way to account for disease severity given the large number of patients missing admission vitals and misdiagnosis was certainly possible. This study identifies the presence of inequalities but does not determine why these inequalities exist, which is an area for future investigation.

\section{Conclusion}

This study demonstrates in this cohort of surgical patients with peritonitis at $\mathrm{KCH}$, Lilongwe, Malawi that men present more frequently for surgical conditions, while women experience more delays accessing surgical care and surgical intervention and have longer hospitalizations than do men. Although the study did not identify sex-based disparities in short-term surgical outcomes, this may be attributable to presentation bias. Further work is needed to determine if sex-based disparities exist in long-term surgical outcomes, including disability or premature death. Future work must focus on the impact of socioeconomic status, age, sex, level of education, healthcare resources and availability on sex-based disparities in access to surgical care. Further research must also evaluate whether sub-optimal clinician-patient communication and unconscious biases lead to sex-based disparities in access to safe and quality surgical care and optimal outcomes. 


\section{References}

1. Whitehead M (1990) The concepts and principles of equity in health. (PDF Report). Copenhagen: WHO, Regional Office for Europe. http://salud.ciee.flacso.org.ar/flacso/optativas/equity_ and_health.pdf Accessed 27 Oct 2017

2. Healthcare quality and disparities in women (2010) The agency for healthcare research and quality. https://archive.ahrq.gov/ research/findings/nhqrdr/nhqrdr10/women.html. Accessed Oct 11 2017

3. Gender and health (2017) UNICEF. https://www.unicef.org/ esaro/7310_Gender_and_health.html. Accessed Oct 112017

4. UN AIDS Prevention GAP Report (2016) Joint united nations programme on HIV/AIDS. www.unaids.org. Accessed Oct 11 2017

5. Khattab H, Yunis N, Zurayk H (1999) Women, reproduction, and health in rural egypt, the giza study. American University Press, Cairo

6. Younis $\mathrm{N}$ et al (1993) A community study of gynecological and related morbidity in rural Egypt. Stud Fam Plan 24(3):175-186

7. Gallaher JR, Cairns B, Varela C, Charles AG (2017) Mortality after peritonitis in sub-Saharan Africa: an issue of access to care. JAMA Surg 152(4):408-410

8. Petroze RT et al (2015) Injury, disability, and access to care in Rwanda: results of a nationwide cross- sectional population study. W J Surg 39(1):62-69. https://doi.org/10.1007/s00268014-2544-9

9. Gale SC, Shafi S, Dombrovskiy VY, Arumugam D, Crystal JS (2014) The public health burden of emergency general surgery in the United States: a 10 year analysis of the Nationwide Inpatient Sample- 2001 to 2010. J Trauma Acute Care Surg 77(2):202-208

10. Tanchangya J, Khan RA, Bayasakh S, Wichaidit W (2015) Gender disparity in delayed treatment-seeking behavior for cataract: 6 years of experience from Impact Jibon Tari Floating Hospital, Bangladesh. Asia Pac J Public Health 27(2):240-247

11. Deery SE, Soden PA, Zetteryall SL et al (2017) Sex differences in mortality and morbidity following repair of intact abdominal aortic aneurysms. J Vasc Surg 65(4):1006-1013

12. Dean WH, Patel D, Sherwin JC, Metcalfe NH (2011) Follow-up survey of cataract surgical coverage and barriers to cataract surgery at Nkhoma, Malawi. Ophthalmic Epidemiol 18(4):171-178

13. Ahmad K, Zwi AB, Tarantola DJ et al (2015) Gendered disparities in quality of cataract surgery in a marginalized population in Pakistan: the Karachi marine fishing communities eye and general health survey. PLoS ONE 10(7):e0131774. https://doi.org/10. 1371/journal.pone.0131774

14. Nkomazana O (2009) Disparity in access to cataract surgical services leads to higher prevalence of blindness in women as compared to men: results of a national survey of visual impairment. Health Care Women Int 30(3):228-229

15. Chhabra ST, Masson S, Kaur T et al (2016) Gender bias in cardiovascular healthcare of a tertiary care centre of north India. Heart Asia 8(1):42-45

16. Bohn JA, Kassaye BM, Record D et al (2016) Demographic and mortality analysis of hospitalized children at a referral hospital in
Addis Ababa, Ethiopia. BMC Pediatr 16(1):168. https://doi.org/ 10.1186/s12887-016-0709-4

17. Nonvignon J, Aikins MK, Chinbuah MA et al (2010) Treatment choices for fevers in children under-five years in a rural Ghanaian district. Malar J 9:188. https://doi.org/10.1186/1475-2875-9-188

18. Bedri H, Romanowski KS, Liao J, Al-Ramahi G, Heard J, Granchi T, Wibbenmeyer L (2017) A national study of the effect of race, socioeconomic status and gender on burn outcomes. J Burn Care Res 38(3):161-168

19. Klitzner TS, Lee M, Rodriguez S, Chang RK (2006) Sex-related disparity in surgical mortality among pediatric patients. Congenit Heart Dis 1(3):77-88

20. Lowry D, Singh J, Mytton J, Tiwara A (2016) Sex-related outcome inequalities in endovascular aneurysm repair. Eur J Endovasc Surg 52(4):518-525

21. Nephew LD, Goldberg DS, Lewis JD, Abt P, Bryan M, Forde KA (2017) Exception points and body size contribute to gender disparity in liver transplantation. Clin Gastroenterol Hepatol 15(8):1286-1293

22. Peracha J, Hayer MK, Sharif A (2016) Gender disparities in living-donor kidney transplant among minority ethnic groups. Exp Clin Transplant 14(2):139-145

23. Hsich EM, Starling RC, Blackstone EH et al (2014) Does the UNOS heart transplant allocation system favor men over women? JAAC Heart Fail 2(4):347-355

24. Bal MM, Saikia B (2007) Gender bias in renal transplantation: are women alone donating kidneys in India? Transplant Proc 39(10):2961-2963

25. Kudenchuk PJ, Maynard C, Martin JS, Wirkus M, Weaver WD (1996) Comparison of presentation, treatment, and outcome of acute myocardial infarction in men versus women (the Myocardial Infarction Triage and Intervention Registry). Am J Cardiol 78:9-14

26. Mahajan K, Negi PC, Merwaha R, Mahajan N, Chauhan V, Asotra S (2017) Gender differences in the management of acute coronary syndrome patients: one year results from the HPIAR (HP-India ACS Registry). Int J Cardiol 248:1-6

27. Worrall-Carter L, McEvedy S, Kuhn L, Scruth E, Maclsaac A, Rahman MA (2017) Systematic review and meta-analysis investigating whether risk stratification explains lower rates of coronary angiography among women with non-ST-segment elevation acute coronary syndrome. J Cardiovasc Nurs 32(2):112-124

28. Du X, Spatz E, Dreyer RP et al (2016) Ex differences in clinical profiles and quality of care among patients with ST-segment elevation myocardial infarction from 2001 to 2011: insights from the China Patient- Centered Evaluative Assessment of Cardiac Events (PEACE)- retrospective study. J Am Heart Assoc 5(2):e002157. https://doi.org/10.14336/ad.2017.1129

29. Zhang B, Zhang W, Huang RC et al (2013) Gender disparities in early death after ST-elevation myocardial infarction. Clin Med J 126(18):3481-3485

30. Goldberg R, Goff D, Cooper L et al (2000) Age and sex differences in presentation of symptoms among patients with acute coronary disease: the REACT Trial. Coron Artery Dis 11:399-407 